\title{
SEQUENTIAL COREFLECTIONS OF STRATIFIABLE SPACES
}

\author{
L. FOGED
}

\begin{abstract}
We prove that the sequential coreflection of a stratifiable space which is
\end{abstract} the union of countably many closed $k$-spaces is stratifiable.

Several years ago E. Michael [10] showed that among spaces which are the union of countably many closed $k$-spaces, the presence of a $\sigma$-locally finite $k$-network allows the preservation of certain topological properties (normality, paracompactness, stratifiability) when forming $k(X)$. In this brief note we show that with use of the Lemma below essentially the same proof will work in $k$-semistratifiable spaces.

Definitions. Let $(X, \mathscr{T})$ be a Hausdorff topological space. A function $S$ : $N \times \mathscr{T} \rightarrow\{A \in \mathscr{P}(x): A$ is closed in $X\}$ is a semistratification [3] of $X$ if the following hold.

(a) If $U \in \mathscr{T}$, then $U=\cup_{n \in \mathrm{N}} S(n, U)$.

(b) If $U \in \mathscr{T}$ and $n \in \mathbf{N}$, then $S(n, U) \subset S(n+1, U)$.

(c) If $U, V \in \mathscr{T}$ with $U \subset V$, and if $n \in \mathbf{N}$, then $S(n, U) \subset S(n, V)$.

A semistratification $S$ of $X$ is said to be a $k$-semistratification [9] of $X$ if:

(d) If $U \in \mathscr{T}$ and $K$ is compact with $K \subset U$, then there is an $n \in \mathbf{N}$ so that $K \subset S(n, U)$; or equivalently:

(d)' If $U \in \mathscr{T}$ and $Z$ is a sequence converging to a member of $U$, then there is an $n \in \mathbf{N}$ so that $S(n, U)$ contains a tail of $Z$.

A collection $\mathscr{F}$ of ordered pairs $F=\left(F_{1}, F_{2}\right)$ of subsets of $X$ with $F_{1} \subset F_{2}$ for all $F \in \mathscr{F}$ is called a pair-cs-network for $X$ if for every open set $U$ of $X$ and a sequence $Z$ converging to a point of $U$, there is an $F \in \mathscr{F}$ so that $F_{2} \subset U$ and $F_{1}$ contains a tail of $Z$. One can show that a space $X$ is $k$-semistratifiable if and only if $X$ has a $\sigma$-cushioned pair-cs-network (cf. $[2,6,8]$ ). Hence any space with a $\sigma$-locally finite $k$-network is $k$-semistratifiable.

Note that because points are $G_{\delta}$ in semistratifiable spaces the notions of sequential space and $k$-space (as well as sequential coreflection and $k$-coreflection) coincide for semistratifiable spaces.

A subset $W$ of a topological space $X$ is said to be a sequential neighborhood of $x \in X$ if every sequence converging to $x$ is eventually in $W$.

Received by the editors August 3, 1983 and, in revised form, November 7, 1983.

1980 Mathematics Subject Classification. Primary 54D50; 54E20. 
LEMMA. Every $k$-semistratifiable space $X$ has a $\sigma$-cushioned pair-cs-network that respects sequential neighborhoods; i.e. if $W$ is a sequential neighborhood of $x$ and $Z$ is a sequence converging to $x$, then there is an $F \in \mathscr{F}$ so that $F_{2} \subset W$ and $F_{1}$ contains a tail of $Z$.

Proof. Let $\mathscr{G}=\bigcup_{n \in \mathrm{N}} \mathscr{G}_{n}$ be a pair-cs-network for $X$, where the families $\mathscr{G}_{n}$ are cushioned. For each $n \in \mathbf{N}$ and $y \in X$, let $U(n, y)$ be an open neighborhood of $y$ so that $U(n, y) \cap \bigcup\left\{G_{1}:\left(G_{1}, G_{2}\right) \in \mathscr{G}_{n}, y \in X \backslash G_{2}\right\}=\varnothing$ and $U(n+1, x) \subset$ $U(n, x)$. For each $W \subset X$ and $n \in \mathbf{N}$, let

$$
F(n, W)=X \backslash \bigcup\{U(n, y): y \in X \backslash W\} .
$$

One may readily verify that for each $n$ the family $\mathscr{F}_{n}=\{(F(n, W), W): W \subset X\}$ is a cushioned family.

To show that $\mathscr{F}=\bigcup_{n \in \mathbf{N}} \mathscr{F}_{n}$ is a pair-cs-network that respects sequential neighborhoods, suppose not. Then there is an $x \in X$, a sequential neighborhood $W$ of $x$, and a sequence $Z=\left\langle x_{k}: k \in \mathbf{N}\right\rangle$ converging to $x$ so that no $F(n, W)$ contains a tail of Z. This allows us to pick a subsequence $\left\langle x_{k(n)}: n \in \mathbf{N}\right\rangle$ so that for all $n, x_{k(n)} \notin$ $F(n, W)$. For every $n \in \mathbf{N}$, there is a $y_{k(n)} \in X \backslash W$ so that $x_{k(n)} \in U\left(n, y_{k(n)}\right)$. We claim that $\left\langle y_{k(n)}: n \in \mathbf{N}\right\rangle$ converges to $x$, violating our assumption that $W$ is a sequential neighborhood of $x$.

Indeed, if $\left\langle y_{k(n)}: n \in \mathbf{N}\right\rangle$ did not converge to $x$, then we could find a neighborhood $V$ of $x$ and a subsequence $\left\langle y_{k(n(m))}: m \in \mathbf{N}\right\rangle$ so that $V \cap\left\{y_{k(n(m))}: m \in \mathbf{N}\right\}=$ $\varnothing$. Find $r \in \mathbf{N}$ and $G \in \mathscr{G}_{r}$ so that $G_{2} \subset V$ and $G_{1}$ contains a tail of $Z$. Then find $m \in \mathbf{N}$ so that $n(m) \geqslant r$ and $x_{k(n(m))} \in G_{1}$. Because $y_{k(n(m))} \notin G_{2}$ we have that $U\left(r, y_{k(n(m))}\right) \cap G_{1}=\varnothing$; thus $U\left(n(m), y_{k(n(m))}\right) \cap G_{1}=\varnothing$, contradicting that $x_{k(n(m))} \in U\left(n(m), y_{k(n(m))}\right)$.

The proof of the following Theorem is essentially that suggested in [10].

THEOREM. If the stratifiable space $X$ is the union of countably many closed $k$-spaces, then $k(X)$ is stratifiable.

Proof. It follows from the Lemma that $k(X)$ has a $k$-semistratification $S$ in which the sets $S(n, U)$ are closed in $X$. Let $D$ be a monotone normality operator [7] for the stratifiable space $X$. We will show that $k(X)$ is also monotonically normal and consequently stratifiable [7, Theorem 2.5]. Write $X=\bigcup_{n \in \mathrm{N}} X_{n}$, where each $X_{n}$ is a closed (in $X$ ) $k$-space. Let $A$ and $B$ be disjoint closed sets in $k(X)$. For every $n$, $A_{n}=A \cap X_{n}$ and $B_{n}=B \cap X_{n}$ are closed in $X_{n}$ and so closed in $X$. Let

$$
U_{n}=\bigcap_{m \in \mathbf{N}} D\left(A_{n} \cup S(m, X \backslash B), B_{m}\right) \backslash B
$$

and

$$
V_{n}=\bigcap_{m \in \mathbf{N}} D\left(B_{n} \cup S(m, X \backslash A), A_{m}\right) \backslash A .
$$

By [10, Lemma 5.3] each $U_{n}$ is open in the subspace $X \backslash B$ of $k(X)$, hence open in $k(X)$. Plainly the $X$-closure of $U_{n}$, and hence the $k(X)$-closure of $U_{n}$, misses $B$. 
Analogous things can be said of the $V_{n}$ 's, so that

$$
U=\bigcup_{n \in \mathrm{N}}\left[U_{n} \backslash \bigcup_{m \leqslant n} \mathrm{cl}_{k(X)} V_{m}\right] \text { and } V=\bigcup_{n \in \mathrm{N}}\left[V_{n} \backslash \bigcup_{m \leqslant n} \mathrm{cl}_{k(X)} U_{m}\right]
$$

are disjoint $k(X)$-open sets containing $A$ and $B$, respectively. Tracing our argument with $A \subset A^{\prime}$ and $B \supset B^{\prime}$ would yield $U \subset U^{\prime}$ and $V \supset V^{\prime}$ and so $k(X)$ is monotonically normal, as desired.

The hypothesis that $X$ be the union of countably many closed $k$-spaces cannot be omitted in the previous Theorem. The space $Z$ of [4] is (as is easily seen) an $M_{1}$-space. So while $Z^{2}$ is $M_{1}, k\left(Z^{2}\right)$ is not regular [4, Theorem C]. ${ }^{1}$ We do not know if "stratifiable" may be replaced by " $M_{1}$ " in our theorem, although it is known that a stratifiable space which is the union of countably many closed metrizable spaces is $M_{1}[5]$.

\section{REFERENCES}

1. C. R. Borges, $A$ stratifiable $k_{R^{-}}$space which is not a $k$-space, Proc. Amer. Math. Soc. 81 (1981), 308-310.

2. J. G. Ceder, Some generalizations of metric spaces, Pacific J. Math. 11 (1961), 105-125.

3. G. D. Creede, Concerning semi-stratifiable spaces, Pacific J. Math. 32 (1970), 47-54.

4. L. O. Foged, Sequential order of homogeneous and product spaces, Topology Proc. 6 (1981), 287-298.

5. G. Gruenhage, On the $M_{3} \rightarrow M_{1}$ question, Topology Proc. 5 (1980), 77-104.

6. J. A. Guthrie, $A$ characterization of $\boldsymbol{\aleph}_{0}$-spaces, Gen. Topology Appl. 1 (1971), 105-110.

7. R. W. Heath, D. J. Lutzer and P. L. Zenor, Monotonically normal spaces, Trans. Amer. Math. Soc. 178 (1973), 481-493.

8. J. A. Kofner, On a new class of spaces and some problems of symmetrizability theory, Soviet Math. Dokl. 10 (1969), 845-848.

9. D. J. Lutzer, Semimetrizable and stratifiable spaces, Gen. Topology Appl. 1 (1971), 43-48.

10. E. Michael, On $k$-spaces, $k_{R^{-}}$spaces and $k(X)$, Pacific J. Math. 47 (1973), 487-498.

Department of Mathematics, University of Texas, El Paso, Texas, 79968

\footnotetext{
${ }^{1}$ Stratifiability was claimed in [1] for an example given in [10] of a $\sigma$-compact cosmic space $X$ so that $k(X)$ is not regular. By our Theorem this claim is incorrect.
} 